\title{
Differential conditionability: Reinforcing grooming in golden hamsters
}

\author{
SAMUEL G. CHARLTON \\ University of New Mexico, Albuquerque, New Mexico
}

\begin{abstract}
Differential conditionability is the empirical finding that not all responses are equally amenable to the same conditioning paradigm. One phenomenon associated with the conditioning of grooming behavior (a difficult-to-condition response) is a decrease in its average duration when followed by food reinforcement. The first experiment investigated this phenomenon by reinforcing golden hamsters (Mesocricetus auratus) with food for grooming or open rearing (a readily conditionable response) under three duration-dependent reinforcement schedules. The obtained data showed that different densities of food delivery had no differential effects on the average durations of grooming responses, indicating that the decreases were not the result of reinforcement-produced interruption. In the second experiment, golden hamsters were reinforced with food for grooming or for open rearing or received free food, under three interval reinforcement schedules. This experiment demonstrated that decreases in the average duration of grooming are independent of grooming behavior's resistance to conditioning. Furthermore, although duration-dependent reinforcement schedules are largely ineffective in conditioning grooming behavior, interval schedules are shown to be quite effective in increasing rates of grooming.
\end{abstract}

At first glance, the prediction and control of grooming behavior appears to be a simple and rather esoteric matter. Its particular importance lies in grooming behavior's particular status as an anomaly for the study of conditioning. Grooming behavior belongs to that class of behaviors that has been called species specific, biologically constrained, and, in some cases, unconditionable. As early as 1911, Thorndike reported difficulty in applying his law of effect to grooming by cats. Instead of increasing like the rates of other instrumental responses receiving reinforcement, the rates of licking and scratching showed, when they increased at all, only small or delayed increases. Subsequently, grooming behavior has been investigated with a variety of experimental paradigms, by a number of different investigators, each arriving at more or less the same conclusion, that grooming behavior does not appear to obey the laws of conditioning. Bolles $(1960) \mathrm{ma}$ nipulated food and water deprivation and exposure to a novel environment in an attempt to modify the incidence of grooming in rats. Bolles reported that some modifications were obtained, but "their nature was too complex to yield any simple expla-

These experiments were conducted as part of a doctoral dissertation to be submitted to the Department of Psychology at the University of New Mexico. The author was supported by NIMH Research Service Award ST32 MH15142-05. The author wishes to thank Douglas P. Ferraro, Jean Newman-Charlton, and the reviewers for their very helpful comments on this manuscript. Requests for reprints should be sent to Samuel G. Charlton, Department of Psychology, University of New Mexico, Albuquerque, New Mexico 87131. nation in terms of controlling stimulus conditions" (p. 310). Bolles and Seelbach (1964) reported that grooming behavior in rats could not be increased with negative reinforcement (noise offset) or be decreased with punishment (noise onset). Shettleworth $(1972,1975,1978)$ reported the failure of both instrumental and classical conditioning paradigms in increasing the rates of face washing in hamsters.

One hypothesis that has been advanced to account for the difficulties surrounding the conditioning of grooming behavior postulates the existence of a hierarchy of naturalistic response classes, each of which is selectively sensitive only to certain functionally relevant stimulus events (Shettleworth, 1975; Walters \& Glazer, 1971). This interpretation states that grooming is difficult to condition because of the intrusion of responses that are functionally relevant to food acquisition and incompatible with grooming. Decreases in the average response duration of grooming produced by response-contingent food delivery are taken as evidence for this incompatibility. Black and Young (1972) offered an alternative hypothesis called the systems constraint hypothesis. This hypothesis suggests that constraints on conditioning naturalistic behavior occur at the level of motivational states rather than at the level of competition between overt responses. Specifically, Black and Young maintain that the conditionability of naturalistic behavior is determined by the extent to which a given response can be brought under the control of contingencies that are not part of the naturally occurring regulatory system or causal agent associated with that response. 
A quite different approach to the problem of differentiating which responses are and which are not conditionable is the temporal and sequential analysis of behavior. As one of the first investigators to take this approach, Bolles (1960) proposed that the sequential relation between grooming and other responses may be the only way to predict the incidence of grooming behavior. Similarly, Staddon (1972) argued that the temporal characteristics of behavior are important determinants of how various responses will be affected by experimental manipulations. In the terminology of this latter analysis, grooming may be classified as an interim response, a behavior that covaries with the reinforcer contingencies while occurring at times when reinforcement is unlikely. Indeed, grooming has been found to increase during the response-contingent reinforcement of other responses (Charlton \& Ferraro, 1982), a finding that supports grooming's classification as an interim response. Shettleworth and Juergensen (1980) applied this analysis to the differential conditionability found with subjects receiving brain stimulation reward. Wall rearing, a terminal behavior that occurred at times when reinforcement was highly probable, was found to be a readily conditionable response.

The present research is directed at expanding our empirical knowledge about grooming behavior in an attempt to accommodate such behavior within the existing principles of behavior. For example, if the temporal patterning of behavior is indeed important in determining the nature of differential conditionability, one promising approach towards prediction and control of these anomalies of conditioning may be based on schedule factors. Inasmuch as schedules of reinforcement determine the exact nature of the temporal relationships between delivery of reinforcement and a variety of responses, it is reasonable to assume that some schedules will be more efficacious than others in conditioning various types of responses. Similarly, a given type of reinforcement contingency may not be universally effective for responses with different topographies. That is, the behavioral effects of rate contingencies, duration contingencies, or temporal contingencies of a reinforcement schedule all depend differentially on the baseline rates, durations, and temporal sequence of various responses. Given the differing topographic features of naturalistic responses, differences typically absent in the arbitrary, or electromechanically defined, responses of general process approaches, it seems plausible that the constraints on the conditioning of grooming behavior may be due to inappropriate reinforcement contingencies. Thus, any conclusions about the constrained or unconditionable nature of various responses may ultimately depend on our knowledge of the interactions between the topographic features of a response and the schedule demands of a given situation.

\section{EXPERIMENT 1}

The purpose of Experiment 1 was to assess the role of reinforcement-produced interruptions of behavior in reducing the average response duration of grooming. As was noted above, one of the anomalous features of grooming behavior is the decrease in the average response duration obtained when grooming is followed by food delivery (Charlton \& Ferraro, 1982; Shettleworth, 1975; Thorndike, 1911). Readily conditionable naturalistic responses, such as open rearing, have not been shown to display any decreases in average response duration when reinforced. This suggests that the decreases in average response duration found in difficult-to-condition responses may be involved in the inability to increase the rates of these responses with reinforcement. In both the Shettleworth and the Charlton and Ferraro investigations, the duration-dependent reinforcement contingencies were such that the response durations of grooming behavior may have been shortened due to the delivery of food soon after the response was begun. Open rearing and other unconstrained responses tend to be of shorter duration, and hence may not have been as affected by reinforcement-produced interruptions.

In the present research, the reinforcement contingency was varied such that, regardless of their response rates, the subjects were exposed to varying reinforcement densities. Thus, if decreases in the duration grooming are due to reinforcementproduced interruption, different densities of reinforcement should differentially decrease grooming's average response duration. In order to compare the effects of this manipulation on grooming with its effects on an unconstrained naturalistic response, a second group of subjects was reinforced for open rearing. The degree to which grooming behavior is constrained because of its incompatibility with food delivery can thus be evaluated in the context of the more general interaction of response duration and reinforcement density.

\section{Method}

Subjects. Ten experimentally naive male golden hamsters (Mesocricetus auratus) were obtained from Simonsen Laboratories, Inc., at approximately 60 days of age. Upon arrival in the laboratory, the hamsters were individually housed in wiremesh cages measuring $18 \times 21.5 \times 38 \mathrm{~cm}$ and always containing nest paper and water. The room in which the hamsters were housed and observed was maintained on a 13-h-light:11-h-dark cycle, with a red photographic safelight illuminated during the dark cycle to facilitate observation. The subjects were food deprived to $90 \%$ of their free-feeding weights for the duration of the experiment. The subjects' daily food ration was determined by their individual weights prior to the daily observation 
session. On days when reinforcement was delivered during the observation session, the subjects' daily food ration was adjusted for the amount of food reinforcement received.

Apparatus. The subjects were observed and conditioned in a Plexiglas open field measuring $61 \times 46 \times 46 \mathrm{~cm}$ and filled to a depth of about $1.5 \mathrm{~cm}$ with wood shavings, an apparatus similar to the open field used by Charlton and Ferraro (1982). The subjects' behavior was recorded by means of a keyboard connected to an on-line H-P $2100 \mathrm{~A}$ minicomputer that recorded the numbers, durations, and sequences of behavior throughout each experimental session. Food reinforcement consisted of $45-\mathrm{mg}$ Noyes food pellets that were mechanically delivered to a clear Plexiglas food cup located in a beveled corner of the apparatus and approximately $0.5-1.0 \mathrm{~cm}$ above the level of the wood shavings.

Procedure. Each subject was conditioned and/or observed for 20 min per day during the first few hours of the dark phase of their light-dark cycle, the time when hamsters are most active (Shettleworth, 1975). During each observation session, the subject's behavior was recorded in terms of 14 mutually exclusive and exhaustive categories. The two responses that were reinforced were: grooming-rubbing forepaws over any part of the body, including interspersed licking of forepaws; open rearing-standing on hind legs with both forepaws free of floor and walls. Ten undergraduate college students were trained to identify the response categories until pairwise interobserver reliability statistics (Kappa coefficients) reached a criterion of $\mathbf{. 8 5}$. Subsequent interobserver reliability tests were conducted at intervals of approximately 2 weeks in order to prevent any divergence of the observers' identification of the response categories. Each subject was observed by a different student each day, every student observing each subject once every 7 days. Thus, differences between observers were distributed such that they could not bias the data in any systematic fashion.

Each subject was randomly assigned to one of two experimental groups. For the subjects assigned to Group 1, food reinforcement was delivered for the performance of grooming; Group 2 received food for the performance of open rearing. Following 7 days of baseline observation, each subject was exposed to three 6-day conditioning phases. The first conditioning phase consisted of food reinforcement delivered on a fixed cumulative duration 2-sec (FCD 2) schedule. This duration-dependent reinforcement schedule delivered a food pellet for every $2 \mathrm{sec}$ that the required response was obtained, regardless of the number of responses necessary to produce the required duration total. The second phase consisted of an FCD 4-sec schedule of food reinforcement, and the third phase of an FCD 8-sec schedule. Following the third conditioning phase, the subjects were observed under extinction conditions for a period of 7 days.

\section{Results and Discussion}

Presented in the top panel of Figure 1 are the average numbers of open rearing responses for Group 1 and the average numbers of grooming responses for Group 2. As can be seen, the rates of responding show a clear differential conditionability effect under all three FCD schedules of food reinforcement. The number of responses for subjects reinforced for open rearing increased dramatically in the presence of food reinforcement, whereas grooming displayed only very slight increases under those same circumstances. There were, however, no substantial differences among the three schedule densities in their effects on the rates of open rearing and grooming. Rates of grooming increased slightly during the first phase (FCD 2) but
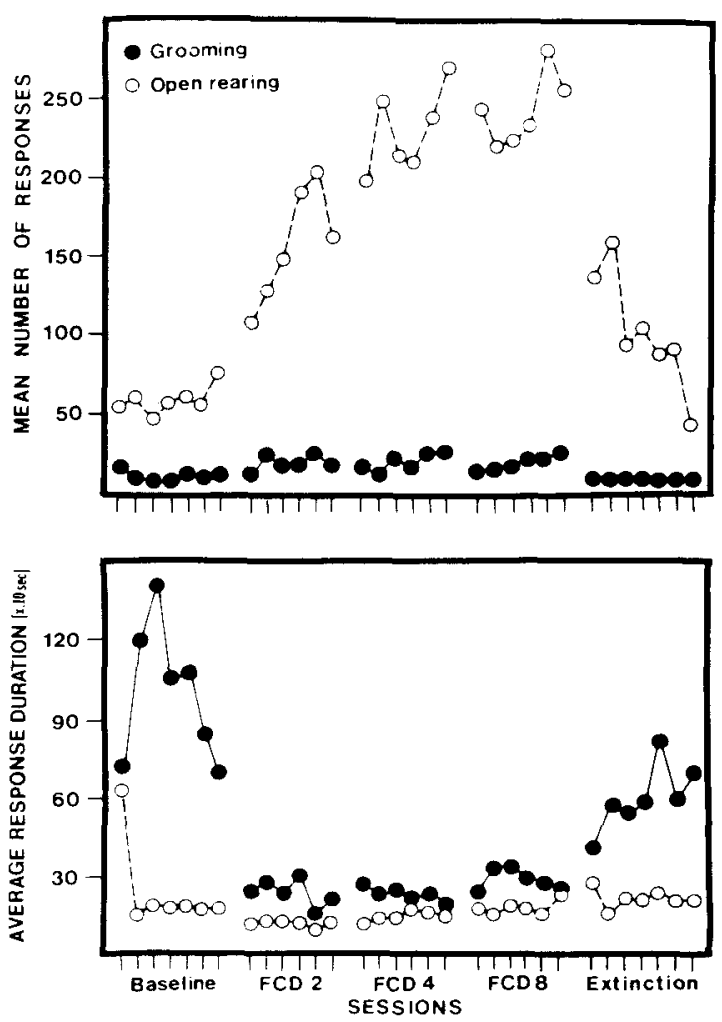

Figure 1. The mean number of responses (top panel) and the average response duration (bottom panel) for grooming and open rearing baseline, FCD 2, FCD 4, FCD 8, and extinction sessions.

did not change appreciably when the reinforcement schedule was switched to FCD 4 and then to FCD 8. Rates of open rearing increased throughout all three conditioning phases. Without a reversal test of the schedule contingencies, it is impossible to tell if the continued increase in rates of open rearing was the result of the different reinforcement schedules, of order effects, or merely of increased practice. Statistical comparison of baseline rates with performance during the final phase of conditioning, the FCD 8 schedule, indicated that the increase in open rearing was highly reliable $[F(1,4)=83.61, p<.01]$. Surprisingly, the slight increases in the number of grooming responses for subjects reinforced for grooming was also significant $[F(1,4)=20.41$, p $<$ $.01]$. Given the relatively small size of the increase in grooming, we may conclude that the significant F statistic obtained for that increase was due to the very low within-subject variability of rates of grooming.

Presented in the lower panel of Figure 1 are the average response durations of open rearing for Group 1 and the average response durations of grooming for Group 2. Marked decreases from baseline durations are evident for grooming during the first conditioning phase, the average duration de- 
creasing from $7 \mathrm{sec}$ on the last day of baseline to $2 \mathrm{sec}$ by the last day of FCD 2 and throughout the remaining reinforcement sessions. The three reinforcement schedules failed to produce any differential suppression of grooming. Open rearing did not display any perceptible changes in duration throughout the experiment. It should be noted that the average response-duration measure is derived from the ratio of rate to total duration. Thus, the decrease in the average response duration of grooming reflects a decrease in the total duration of grooming obtained, because there were no changes in rates commensurate with the decreases in the average duration of grooming. Statistical analysis indicated that the decreases in the durations of grooming, when baseline was compared with performance under the FCD 8 schedule, were highly reliable $[F(1,4)=30.00, p<$ $.01]$, whereas there was no statistical difference between baseline and conditioning for the average duration of open rearing $[F(1,4)=.06, p>.05]$.

These results correspond very well to previous reports of differential conditionability (Charlton \& Ferraro, 1982; Shettleworth, 1975) in that rates of grooming were found to resist substantial modification via response-contingent food delivery, while the average response durations of grooming actually decreased when grooming was followed by food delivery, returning to baseline levels during extinction. In contrast, the rate of open rearing was found to increase sharply when open rearing was followed by food delivery, although the average duration remained unaffected. In the context of the present experiment, however, these effects were rather disappointing in that there were no appreciable differences in the effects of the three different densities of food reinforcement upon either the rate or duration of grooming behavior. The finding of a decrease in the average response duration of grooming even during the FCD 4 and FCD 8 schedules tends to discount the role of reinforcement-produced interruptions in accounting for either the decreases in the average response durations of grooming or the relatively small increases in rates of grooming when grooming was followed by food delivery.

Analysis of the sequential relationships between various responses during conditioning, however, provided further information with which to evaluate the role of reinforcement-produced interruptions. Presented in Figure 2 are the probabilities of grooming and open rearing for the $8 \mathrm{sec}$ preceding and following food delivery during the FCD 8 reinforcement schedule. As can be seen in Figure 2, the probability of open rearing increases gradually in the time preceding food delivery, decreases greatly immediately after food delivery, and increases gradually thereafter. The probability of grooming, on the other hand, increases just prior to food delivery and

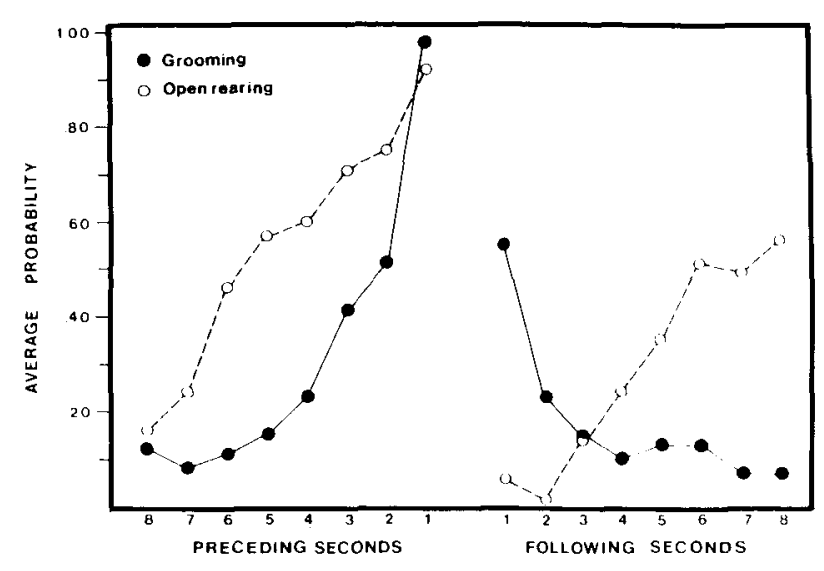

Figure 2. The average response probabilities of grooming and open rearing for the 8 sec preceding and following reinforcement on the FCD 8 schedule.

gradually decreases after food delivery. Performance of open rearing appears appropriate to the cumulative-duration contingency, whereas the probabilities of grooming are indicative of relatively poorer adaptation to the schedule of reinforcement. The rather long latency between food delivery and the termination of grooming suggests either a lack of sensitivity to food delivery or a resistance to reinforcement-produced interruption. The decreasing probability of grooming in the time following food delivery is representative of the low rates of grooming obtained with the duration-dependent reinforcement schedule. Furthermore, this analysis reveals that food delivery did not prevent the subjects from grooming, which indicates that the decreases in the average response duration of grooming were not solely the result of reinforcement-produced interruption.

These data portray grooming as a response that is performed at relatively low rates that increase only slightly in the presence of food reinforcement. Furthermore, once initiated, grooming is not quickly terminated in response to food delivery. The question of whether the difficulties in increasing rates of grooming are due to the decreases in the average response duration is still not definitively answered. We can be reasonably sure, however, that it is not merely the density of reinforcement-produced interruptions that is responsible for the decrease in the average response duration. An alternative interpretation of these findings is that the cumulative duration schedule of reinforcement favors responses with short average response durations (e.g., open rearing). Responses of longer average durations may be difficult to condition simply because of the difficulty in matching response durations to the schedule demands. Each of the reinforcement contingencies $(2,4$, or $8 \mathrm{sec})$ was shorter than or nearly 
equal to the average response duration of grooming during baseline, for which there were no daily average durations less than $7 \mathrm{sec}$. In comparison, the average response duration of open rearing during baseline was approximately $2 \mathrm{sec}$, and hence was not as frequently interrupted by reinforcement. The second experiment in this series pursued the latter interpretation by utilizing a temporal rather than a duration-dependent reinforcement contingency.

\section{EXPERIMENT 2}

In the following experiment, further analysis of schedule factors was directed at investigating the extent to which decreases in the average response duration are responsible for the constraints on conditioning grooming with food reinforcement. As was stated above, increasing the reinforcement density did not prevent decreases in the average response duration of grooming. It was suggested that given the nature of duration-dependent reinforcement schedules, responses with short response durations (e.g., open rearing) are favored over responses of long duration (e.g., grooming) in their adaptability to the schedule demands. In order to examine this possibility, a series of temporal reinforcement schedules was selected so that no explicit change in the duration of grooming was required to meet the reinforcement contingency. To provide an example of a relatively unconstrained naturalistic response, a second group of subjects was reinforced for open rearing with the same contingency. As a further control, and in order to evaluate the temporal patterning of behavior in response to food delivery, another group of subjects was exposed to a series of noncontingent reinforcement schedules.

It was predicted that the temporal schedules would serve to remove any differential conditionability effects that were solely the result of differences in the average response durations of open rearing and grooming. If, however, rates of grooming were observed to increase in the presence of decreases in average response duration, we could conclude that decreases in average response duration were not responsible for the difficulties in increasing the rates of grooming. Furthermore, even if rates of grooming were found to be constrained or invariant, it might have been possible for subjects to distribute their time spent grooming so as to maximize food delivery. Finally, if the temporal order, or sequential nature, of a response was a constraint on the conditionability of naturalistic responses, then a reinforcement schedule with an inherent temporal regularity might have elucidated the nature of those constraints.

\section{Method}

Subjects. Ten experimentally naive male golden hamsters (Mesocricetus auratus) were obtained from Simonsen Laboratories, Inc., at approximately 60 days of age. Upon arrival, these subjects were housed and fed in the same manner as the subjects in Experiment 1.

Apparatus. The open field and recording apparatus described in Experiment 1 were again used for Experiment 2.

Procedure. A second group of 10 undergraduate college students were trained as observers for Experiment 2. The response categories, interobserver reliability tests, rotation of observers across subjects, and daily session length remained the same as in Experiment 1. Each subject was assigned to one of three experimental groups. The four subjects assigned to Group 1 were reinforced for grooming, the three subjects in Group 2 were reinforced for open rearing, and the three subjects assigned to Group 3 received noncontingent food. Following 10 days of baseline observation, each subject was exposed to three successive reinforcement phases. During the first phase, the subjects in Groups 1 and 2 were reinforced for 18 days on a fixed interval 60-sec (FI 60) schedule, and the subjects in Group 3 received free food on a fixed time 60-sec (FT 60) schedule. During the second conditioning phase, the FI and FT contingencies were changed to $30 \mathrm{sec}$ for 10 days and then to $8 \mathrm{sec}$ for 10 days in the third phase. Following the third reinforcement phase, the subjects were observed under extinction conditions for a period of 5 days.

\section{Results and Discussion}

Presented in the upper panel of Figure 3 are the average numbers of grooming responses for the subjects in Groups 1 and 3 (subjects receiving reinforcement for grooming and noncontingent food delivery,
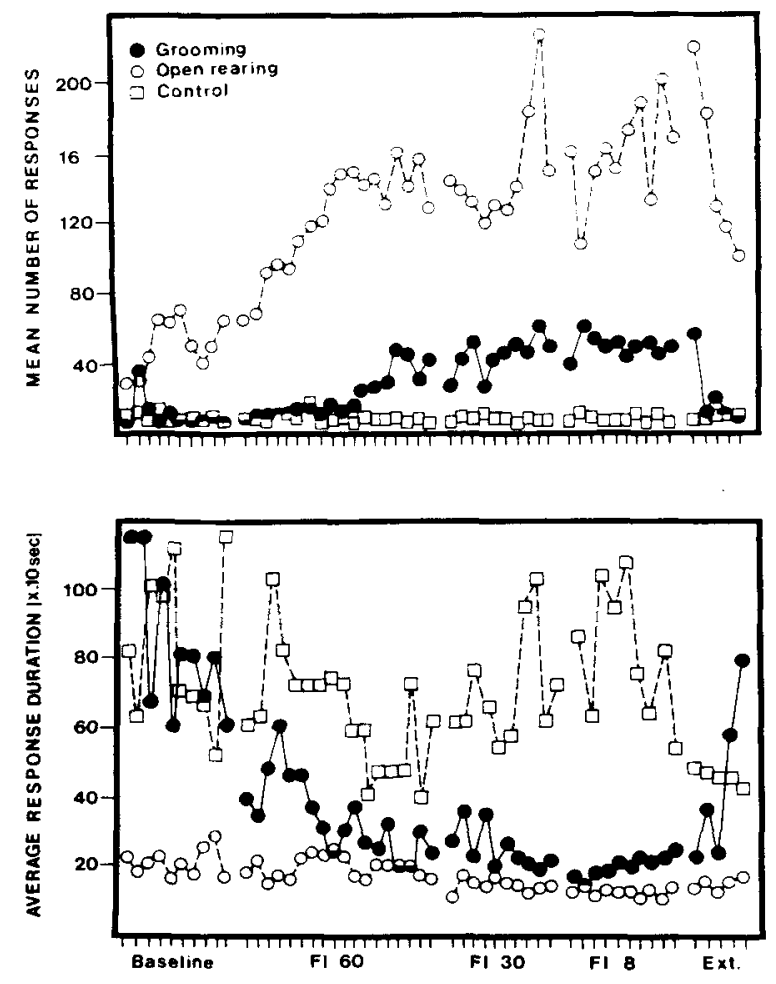

Figure 3. The mean number of responses (top panel) and the average response duration (bottom panel) for grooming and open rearing during baseline, FI 60, FI 30, FI 8, and extinction sessions. Also presented are the rates (top panel) and average durations (bottom panel) for grooming by control subjects during baseline, FT 60, FT 30, FT 8, and extinction sesslons. 
respectively), as well as the average number of open rearing responses for the subjects in Group 2 (reinforcement of open rearing). Figure 3 shows that the FI reinforcement schedules increased the rates of grooming, relative to both baseline levels and subjects receiving noncontingent food. The difference in the rates of grooming obtained for Groups 1 and 3 indicates that the increase during FI reinforcement was not the result of the reactive effects of food delivery itself. The figure also shows that rates of open rearing were significantly increased by the FI reinforcement schedules. This finding is important in assuring that by unconstraining the conditioning of grooming, we have not inadvertently constrained a previously conditionable response, open rearing. It should be noted that there is still a differential conditionability effect in that open rearing reaches higher levels than does grooming. Given the initial differences in the baseline rates of the two responses, this finding is not surprising. This fact, coupled with the clear decrease in grooming obtained when FCD 8 was changed to extinction, is a good indication that grooming was effectively conditioned. Finally, it can be seen that there were no differential effects of the three FI schedules upon the rates of either open rearing and grooming. Statistical comparison of rates during the FI 8 schedule with baseline rates indicated that the increases were statistically significant $[F(1,3)=31.66, p<.01$, and $F(1,2)=52.14, p<.01$, for grooming and open rearing, respectively].

The average response durations of open rearing for Group 2 and of grooming for Groups 1 and 3 are displayed in the lower panel of Figure 3. Here it can be seen that the abbreviation of grooming responses produced by response-contingent reinforcement is still obtained, even though the FI schedule significantly increased the subjects' rates of grooming. Similarly, the average response durations of open rearing, previously unaffected by reinforcement, are now somewhat reduced by the third reinforcement phase. The average response duration of grooming by subjects receiving noncontingent food, however, did not display any decreases due to reinforcement. This latter finding indicates that it is indeed the presence of a response contingency that is responsible for decreases in average response duration. As with the rate measure, the average response duration measure did not display any differential effects of the three reinforcement densities. The statistical comparison of baseline with performance on the FI 8 indicated a significant decrease in the average response durations of grooming for the subjects in Group $1[F(1,3)=66.86, p<.01]$ and of open rearing for the subjects in Group $2[F(1,2)=16.71$, $\mathrm{p}<.01]$, but not in grooming for subjects in Group 3 $[\mathrm{F}(1,2)=.32, \mathrm{p}>.05]$.

The significant decrease in the average response duration of open rearing is an unexpected finding.
It suggests that FI reinforcement schedules produce decreases in the average response duration of open rearing similar to those produced in grooming by either FCD or FI schedules. As verification of this, the sequential probabilities of open rearing and grooming, for the $8 \mathrm{sec}$ preceding and the $8 \mathrm{sec}$ following reinforcement on the FI 8 schedule, are presented in Figure 4. For the sake of clarity, the probabilities for the control subjects were omitted from this figure, there being no systematic temporal distribution of grooming under the FT 8 schedule. As was suggested by the average duration data, open rearing and grooming now appear very similar in probability during the time surrounding reinforcement. Both responses show large increases in the seconds immediately preceding reinforcement, and a persistence of responding in the seconds immediately following reinforcement. Most importantly, this finding demonstrates that decreases in response duration produced by conditioning are not limited to grooming or other constrained responses. In the present experiment, the FI schedules delivered reinforcement, when appropriate to the interval, as soon as a response occurred. Delivery of reinforcement was unrelated to differing response durations, thus making open rearing and grooming equally likely to be interrupted. As a consequence, significant decreases in average response durations were found for both open rearing and grooming under FI reinforcement schedules.

These results suggest a number of things: First, decreases in average response duration are not responsible for the difficulties associated with the conditioning of grooming. The decreases in the average response duration of grooming were obtained with both the FCD and FI schedules, but did not prevent grooming from increasing in rate during the FI schedule. Second, the absence of any observable decreases in the average response duration of groom-

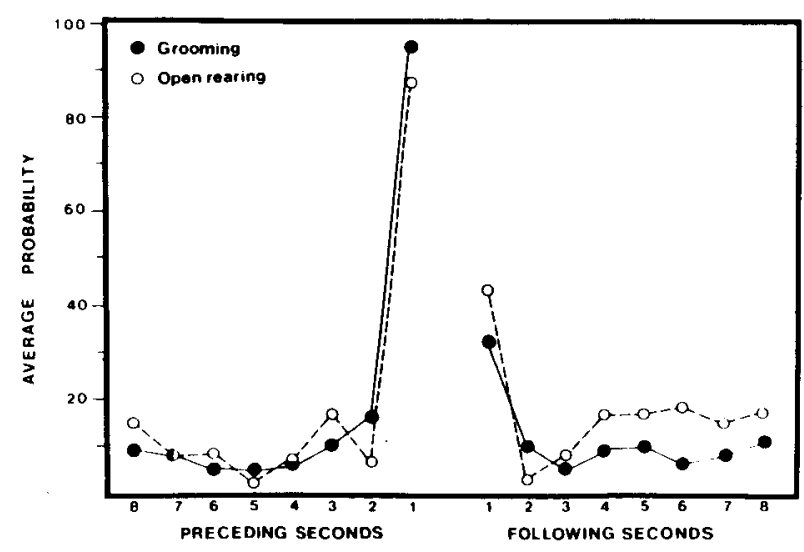

Figure 4. The average response probabillties of grooming and open rearing for the 8 sec preceding and following reinforcement on the FI 8 schedule. 
ing by subjects receiving free food suggests that it is indeed the presence of a response contingency that is responsible for decreases in average response duration. Finally, the reliable increases in rates of grooming obtained with FI reinforcement schedules indicates that grooming does not represent a special class of unconditionable behavior. The finding of decreased response durations of open rearing, in conjunction with increased rates due to FI reinforcement, is strong evidence for the proposition that conditioning grooming is similar to conditioning other naturalistic processes.

\section{GENERAL DISCUSSION}

These experiments demonstrate a number of important effects associated with the conditioning of grooming behavior. First and foremost, decreases in the average response duration of grooming are not responsible for grooming behavior's resistance to conditioning. The increased rates of grooming observed under the FI schedules were apparently independent of the decreases in duration obtained under those same contingencies. Furthermore, the observation that open rearing also decreases in average duration indicates that such decreases are not unique to constrained behaviors. Second, decreases in average response duration are not solely the result of reinforcement-produced interruption. Different densities of reinforcement had no differential effects on the average response duration of grooming, as should have been the case if the decreases were produced by the delivery of reinforcement. Similarly, the average response-probability data indicated that grooming was not reliably interrupted by food delivery, since the subjects continued to groom for up to $3 \mathrm{sec}$ after food was delivered.

Finally, grooming is not representative of any class of constrained or unconditionable behaviors. Reliable increases in rates of grooming were obtained under the FI reinforcement schedules. The differential efficacy of FCD and FI schedules in increasing rates of grooming is presumed to be the result of temporal characteristics of the FI schedules that are absent in FCD schedules. Another potential difference between the two types of schedules is the differential reinforcement density associated with each schedule. In the present experiments, however, density of reinforcement rarely had any effects on the rates or durations of grooming. Furthermore, the FCD 8 schedule used in Experiment 1 enabled a reinforcement density identical to that of the FI 8 schedule used in Experiment 2, yet the differential rates of grooming obtained with these two schedules discounts the role of reinforcement density in the differential success of the two schedules.

One issue especially pertinent to the present research is that of response definition. Earlier inves- tigations of differential conditionability (Charlton \& Ferraro, 1982; Shettleworth, 1975) have focused on face washing, one component of the more molar grooming response discussed here. Grooming behavior, as defined in the present investigation, includes side and belly washing as well as face washing. Inasmuch as side and belly washing nearly always follows face washing in a bout of grooming, these topographically similar responses were treated as a single functional unit (i.e., grooming) in the present research. Thus, side and belly washing, as defined by Shettleworth (1975) and others, is approximately equivalent to long-duration grooming responses, whereas face washing is equivalent to shortduration grooming in the present research. Annable and Wearden (1979) reported that body washing (side and belly washing) by rats, but not face washing, could be increased with food reinforcement. In the present investigation, however, there were no differential effects of the long- or short-duration FCD schedules upon either long- or short-duration grooming responses. Furthermore, observation of the subjects in Experiment 2 revealed that the increased grooming produced by the FI schedules almost always took the form of face washing (shortduration grooming) rather than body washing. Therefore, although the implications of classifying grooming as a single response versus two or more response classes are not resolved by the present investigation, increases in the rates of grooming are not the result of increases in a more readily conditionable body washing response.

Let us return now to the hypotheses advanced to account for grooming behavior's resistance to conditioning. Sequential analysis indicated that the decrease in the average response duration of grooming produced by reinforcement was the result of reinforcement-produced interruption and was not due to any incompatibility of grooming and food anticipation. Furthermore, it was found that the decrease in average response duration was not the primary difficulty in conditioning grooming, the decrease being present even when rates of grooming were increased with the FI reinforcement schedule. Moreover, the decrease in the average duration of open rearing found in Experiment 2 indicates that the decreased duration is not a phenomenon associated solely with constrained responses like grooming. The decrease may be obtained whenever the reinforcement schedule does not explicitly allow for the average duration of the referent response. This effect is typically absent in experiments involving the conditioning of responses such as leverpressing and keypecking by virtue of their definition as electromechanical switch closures. Naturalistic responses that can vary in duration, as well as in rate, are subject to interactions with reinforcement contingencies that 
are not found with arbitrary, experimentally defined responses.

Taken together, the results of these experiments are a strong indication that grooming does not represent a special class of constrained behavior. To be sure, differences between various responses do exist, as can be seen in the differences between open rearing and grooming obtained in the preceding experiments. These differences in conditionability do not, however, represent species-specific or responsespecific constraints that are beyond the level of a general process analysis. Instead, the existence of differential conditionability among various responses is more likely the result of unforeseen interactions between schedule contingencies and differing response topographies. It is the opinion of this author that these differences in conditionability represent only differences in degree, rather than qualitative differences among classes or types of behavior. Furthermore, it is suggested that the differential conditionability phenomenon is subject to lawful and orderly systematization and prediction, and as such can be integrated into the existing principles of conditioning. To this end, the systematic investigation of the differential conditionability of behavior may be more productive than the study of isolated constraints on the laws of condtioning.

\section{REFERENCES}

Annable, A., \& Wearden, J. H. Grooming movements as operants in the rat. Journal of the Experimental Analysis of Behavior, 1979, 32, 297-304.

Black, A. H., \& Young, G. A. Constraints on the operant conditioning of drinking. In R. M. Gilbert \& J. R. Millenson
(Eds.), Reinforcement: Behavioral analyses. New York: Academic Press, 1972.

Bolles, R. C. Grooming behavior in the rat. Journal of Comparative and Physiological Psychology, 1960, 53, 306-310.

Bolles, R. C., \& Seelbach, S. Punishing and reinforcing effects of noise onset and termination for different responses. Journal of Comparative and Physiological Psychology, 1964, 58, 127-131.

Charlton, S. G., \& Ferraro, D. P. Effects of deprivation on the differential conditionability of behaviour in golden hamsters. Experimental Animal Behaviour, 1982, 1, 18-29.

Shetrleworth, S. J. Food reinforcement and the organization of behavior in golden hamsters. In R. A. Hinde \& J. S. Hinde (Eds.), Constraints on learning. New York: Academic Press, 1972.

ShetTLE WORTH, S. J. Reinforcement and the organization of behavior in golden hamsters: Hunger, environment, and food reinforcement. Journal of Experimental Psychology: Animal Behavior Processes, 1975, 1, 56-87.

Shettleworth, S. J. Reinforcement and the organization of behavior in golden hamsters: Pavlovian conditioning with food and shock unconditioned stimuli. Journal of Experimental Psychology: Animal Behavior Processes, 1978, 4, 152-169.

Shettleworth, S. J., \& Jueraensen, M. R. Reinforcement and the organization of behavior in golden hamsters: Brain stimulation reinforcement for seven action patterns. Journal of Experimental Psychology: Animal Behavior Processes, 1980, 6, 352-375.

Staddon, J. E. R. Temporal control and the theory of reinforcement schedules. In R. M. Gilbert \& J. R. Millenson (Eds.), Reinforcement: Behavioral analyses. New York: Academic Press, 1972.

ThORNDike, E. L. Animal intelligence. New York: Macmillan, 1911.

Walters, G. C., \& Glazer, R. D. Punishment of instinctive behavior in the Mongolian gerbil. Journal of Comparative and Physiological Psychology, 1971, 75, 331-340.

(Manuscript received August 6, 1982; revision accepted for publication December 30,1982 .) 